\title{
Cornucopia of Antineutrons and Hyperons from a Super $J / \psi$ Factory for Next-Generation Nuclear and Particle Physics High-Precision Experiments
}

\author{
Chang-Zheng Yuan $\oplus^{1,2, *}$ and Marek Karliner $\circledast^{3, \dagger}$ \\ ${ }^{1}$ Institute of High Energy Physics, Chinese Academy of Sciences, Beijing 100049, China \\ ${ }^{2}$ University of Chinese Academy of Sciences, Beijing 100049, China \\ ${ }^{3}$ School of Physics and Astronomy, Tel Aviv University, Tel Aviv 69978, Israel
}

(Received 13 April 2021; revised 7 May 2021; accepted 26 May 2021; published 30 June 2021)

\begin{abstract}
In order to study the interactions and structure of various types of matter, one typically needs to carry out scattering experiments utilizing many different particles as projectiles. Whereas beams of $e^{ \pm}, \mu^{ \pm}, \pi^{ \pm}, K^{ \pm}$, protons, antiprotons, and various heavy ions have been produced and have enabled many scientific breakthroughs, beams of antineutrons, hyperons $(\Lambda, \Sigma$, and $\Xi)$ and their antiparticles are typically not easy to obtain. Here we point out and investigate a new high-quality source of these particles: a super- $J / \psi$ factory with the capability of accumulating trillions of $J / \psi$ decays each year. In the relevant $J / \psi$ decays, the desired particle is produced together with other final-state particles that can be tagged. This allows accurate determination of the flux and momentum of the projectile, enabling unprecedented precision in the study of the corresponding interactions with a broad range of targets. These novel high-statistics sources of baryons and antibaryons with precisely known kinematics open fresh opportunities for applications in particle and nuclear physics, including antinucleon-nucleon interaction, a nonvalence $s \bar{s}$ component of the nucleon, (anti) hyperon-nucleon interaction, OZI violation, (multistrange) hypernuclei, exotic light hadron spectroscopy, and many others, as well as calibration of Monte Carlo simulation for hadronic and medical physics.
\end{abstract}

DOI: 10.1103/PhysRevLett.127.012003

Scattering experiments using many different kinds of beams are the mainstay of experiments investigating the fundamental interactions and structure of matter at the subatomic level. Beams of long-lived charged particles and of photons are easy to produce, and so many experiments using charged projectiles have been carried out during the more than 100 years since the trailblazing experiment shooting $\alpha$ particles into gold foil that enabled Rutherford to infer the existence of the atomic nucleus [1]. Since then, $e^{ \pm}, \mu^{ \pm}, \pi^{ \pm}, K^{ \pm}$, proton, antiproton, photon, and various heavy ion beams have been produced and have served as enablers of many scientific breakthroughs. Beams of some neutral particles, like neutrons and neutrinos, are relatively easy to produce but difficult to control; i.e., they have a large momentum spread. Beams of other neutral particles, such as antineutrons and $K^{0} / \bar{K}^{0}$, and of long-lived hyperons $\left(\Lambda, \Sigma^{+,-}, \Xi^{0,-}\right)$ and their antiparticles $\left(\bar{\Lambda}, \bar{\Sigma}^{-,+}, \bar{\Xi}^{0,+}\right)$ have great physics potential, but they are typically much more difficult to produce and control.

Although potentially extremely useful for investigating nonperturbative QCD and nuclear structure, experimental

Published by the American Physical Society under the terms of the Creative Commons Attribution 4.0 International license. Further distribution of this work must maintain attribution to the author(s) and the published article's title, journal citation, and DOI. Funded by SCOAP. studies using antineutron beams have been very limited till now, due to the severe difficulties in accumulating a sufficient number of antineutrons with known flux and momentum [2]. The best antineutron sources so far have been obtained at BNL E-767 [3] (with momentum between 100 and $500 \mathrm{MeV} / c$ ) and the CERN OBELIX experiments [4] (with momentum between 50 and $400 \mathrm{MeV} / c$ ), enabling quite a wide range of physics topics to be studied, from nuclear physics to hadron spectroscopy, albeit with limited statistics. The scattering of antineutrons on nuclei made it possible to investigate nucleon-antinucleon annihilation inside matter without complications due to Coulomb interaction [2].

In the above experiments, antineutrons were produced in proton-antiproton annihilation via charge exchange (CEX) $\bar{p} p \rightarrow \bar{n} n$. The disadvantages of this method are obvious: the production rate is low, and the antineutron momentum and direction are hard to control. The selection of antineutrons with momentum in a specific direction results in discarding a large fraction of antineutrons. Further higherrate experiments have been proposed, utilizing highintensity antiproton beams, but within this approach, one does not expect a significant improvement in the antineutron accumulation rate.

Ground-state $S U(3)$ octet hyperons $\left(\Lambda, \Sigma^{+,-}, \Xi^{0,-}\right)$ and their antiparticles have relatively long lifetimes (with a typical $c \tau$ of a few centimeters) and are essential for 
investigating several important physics questions, including hyperon-nucleon interaction and and the possible role of hyperons in neutron stars [5-7]. The relevant experimental studies started in the 1960s and have lasted for more than half a century [8-17], using $\pi^{ \pm}$or $K^{ \pm}$beams shot into bubble chambers or scintillating fiber (SciFi) targets. The statistics of these experiments are low, with typically a few tens to a few hundreds of observed events.

In this Letter, we propose a new source of antineutrons and (anti)hyperons, based on a completely different technique. Due to its large production cross section in $e^{+} e^{-}$and large decay branching fractions to final states with antineutrons or (anti)hyperons, $J / \psi$ particles produced in an $e^{+} e^{-}$annihilation experiment can serve as a new source of antineutrons and (anti)hyperons. By tagging the particles recoiling against an antineutron or (anti)hyperon in $J / \psi$ decays, the momentum and direction of these source particles can be determined precisely, and the interaction with the target material placed outside of a very smallradius $e^{+} e^{-}$beam pipe allows substantial high-precision measurements related to particle and nuclear physics.

We show below that antineutrons produced in $J / \psi \rightarrow p \pi^{-} \bar{n}$ and (anti)hyperons produced in similar $J / \psi$ decay modes at a tau-charm factory like BESIII [18] at BEPCII [19], or a super-tau-charm factory like STCF [20] or SCTF [21], can serve as a perfect source of these particles for many physics studies, thanks to the huge $e^{+} e^{-} \rightarrow J / \psi$ production rate and the modern, multipurpose high-performance detectors. We use the existing BESIII experiment as a case study, with its accumulated dataset of $1 \times 10^{10} \mathrm{~J} / \psi$ 's, to provide a proof of concept of using antineutrons from $J / \psi$ decays. We extend our discussion to $S U(3)$ octet hyperon and antihyperon sources and to future higher-luminosity experiments under consideration. We point out that by including in the detector design an option for inserting a variety of specific materials as targets for these particles, a wide range of novel highprecision physics measurements can be carried out. These include antinucleon-nucleon interaction [22], OZI violation, nonvalence $s \bar{s}$ components of the nucleon [23-25], (anti)hyperon-nucleon interaction [5,7], (multi-strange) hypernuclei [26-28], light hadron spectroscopy [29,30], including exotics and many others [2], as well as cross sections of antineutrons with material for the calibration of Monte Carlo simulation codes for particle physics and medical applications, such as FLUKA [31] and GEANT4 [32].

The $J / \psi$ meson was discovered in $1974[33,34]$ and has been studied in many generations of detectors. Due to its large partial width to $e^{+} e^{-}$, the production cross section of $J / \psi$ in $e^{+} e^{-}$annihilation is about $90 \mu \mathrm{b}$. However, the actual production rate suffers from initial-state radiation and the energy spread of the $e^{+} e^{-}$beams [35]. As a matter of fact, the experimental production cross section of $J / \psi$ at BEPCII is $3500 \mathrm{nb}$ with an energy spread of $0.9 \mathrm{MeV}$ for the center-of-mass energy [36]. The BEPCII [19] is a symmetric $e^{+} e^{-}$collider operating in center-of-mass energies from 2 to $5 \mathrm{GeV}$, with a design luminosity of $10^{33} \mathrm{~cm}^{-2} \mathrm{~s}^{-1}$ at $3.77 \mathrm{GeV}$, the peak of the $\psi(3770)$ resonance. The luminosity at $3.097 \mathrm{GeV}$, the $J / \psi$ resonance, is about $0.47 \times 10^{33} \mathrm{~cm}^{-2} \mathrm{~s}^{-1}$. The energies of the $e^{+}$and $e^{-}$beams are known to $0.1 \mathrm{MeV}$, and they collide with a crossing angle of $22 \mathrm{mrad}$ in the horizontal plane, so the $J / \psi$ particle is basically static with a very small boost, which is known precisely. This is equivalent to a production rate of $1600 \mathrm{~Hz}$ for $J / \psi$ events.

Among more than 300 decay modes reported, $J / \psi \rightarrow$ $p \pi^{-} \bar{n}$ is the best source of antineutrons: the branching fraction, $(2.12 \pm 0.09) \times 10^{-3}$, is large [37], and there are only two charged tracks originating from the interaction point. They can be selected and identified with high efficiency in a detector like BESIII.

The BESIII detector is described in detail in Ref. [18]. The cylindrical core of the detector covers $93 \%$ of the full solid angle and consists of a helium-based multilayer drift chamber (MDC) outside of a beryllium beam pipe, a plastic scintillator time-of-flight system (TOF), and a CsI(Tl) electromagnetic calorimeter (EMC), which are all enclosed in a superconducting solenoidal magnet providing a $1.0 \mathrm{~T}$ magnetic field. The solenoid is supported by an octagonal flux-return yoke with resistive plate counter muon identification modules interleaved with steel. The chargedparticle momentum resolution at $1 \mathrm{GeV} / c$ is $0.5 \%$, and the $d E / d x$ resolution is $6 \%$ for electrons from Bhabha scattering. The EMC measures photon energies with a resolution of $2.5 \%(5 \%)$ at $1 \mathrm{GeV}$ in the barrel (end cap) region. The time resolution in the TOF barrel region is $68 \mathrm{ps}$, while that in the end cap region is $110 \mathrm{ps}$. The end cap TOF system was upgraded in 2015 using multigap resistive plate chamber technology, providing a time resolution of 60 ps [38]. In four runs in 2009, 2012, 2018, and 2019, BESIII has accumulated $1 \times 10^{10}$ highquality $J / \psi$ events. These have yielded a large number of significant published results, based on a part of or the full data sample [18].

With known four-momenta of the initial $J / \psi$ and those of the selected proton and $\pi^{-}$, the antineutron can be selected by requiring the recoiling mass of $p \pi^{-}$to agree with that of an antineutron. The momentum and direction of the antineutron can be determined as well, with uncertainties of a few $\mathrm{MeV}$ and a few milliradians, respectively. This estimate is based on published results on $J / \psi \rightarrow p \pi^{-} \bar{n}$ from BES [39], whose momentum resolution is not as good as that of BESIII, and $\psi(2 S) \rightarrow \gamma \chi_{c J} \rightarrow \gamma p \pi^{-} \bar{n}$ from BESIII [40], where one more photon is involved in the final state. With $\mathcal{B}\left(J / \psi \rightarrow p \pi^{-} \bar{n}\right) \approx 2 \times 10^{-3}$, the $\sim 40 \%$ tagging efficiency indicates that $8 \times 10^{6}$ antineutrons can be tagged in the $1 \times 10^{10} \mathrm{~J} / \psi$ event sample.

The maximum momentum of the antineutron is $1174 \mathrm{MeV} / c$, corresponding to the case when the proton and $\pi^{-}$fly in the same direction, opposite to the 

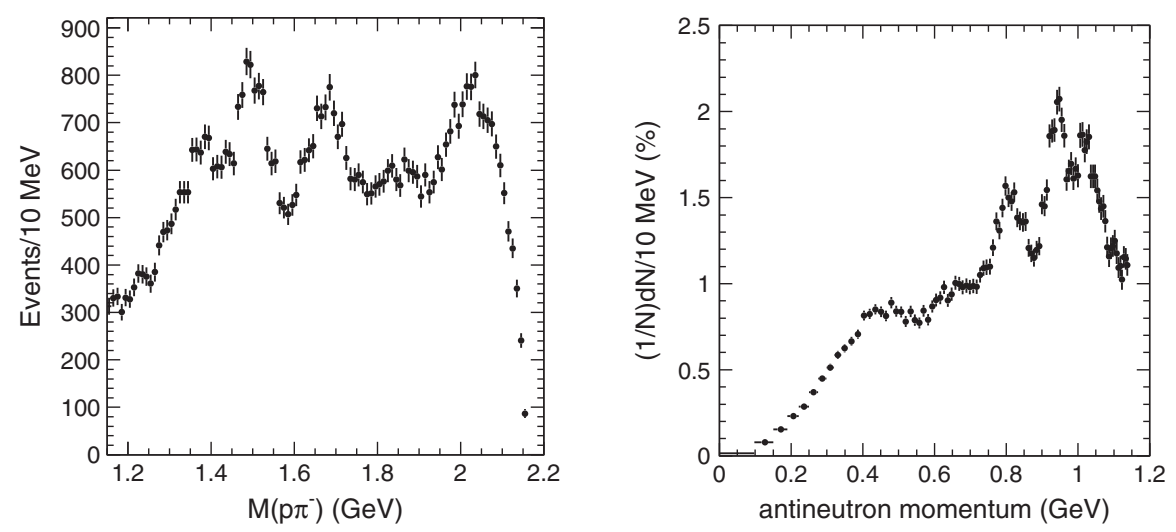

FIG. 1. Invariant mass distribution of $p \pi^{-}$from $J / \psi \rightarrow p \pi^{-} \bar{n}$ selected from the BES experiment (left), and the corresponding antineutron momentum distribution (fraction of events in $10 \mathrm{MeV} / c$ ) (right). The plots have been remade with data in Ref. [39].

antineutron; the minimum momentum of the antineutron is zero, when the proton and $\pi^{-}$fly back-to-back with the same absolute value of the momentum. The momentum spectrum of the antineutron, shown in the right panel of Fig. 1, is determined by the $J / \psi \rightarrow p \pi^{-} \bar{n}$ decay dynamics. It can be obtained by converting the $p \pi^{-}$invariant mass distribution [39] (shown in the left panel of Fig. 1) into antineutron momentum distribution.

Comparing this sample with that achieved by the OBELIX experiment [41], we find that the BESIII sample is already a quarter of that in OBELIX, and the momentum range is wider. The momentum of the antineutron at BESIII is known with an uncertainty of about $6-7 \mathrm{MeV} / c$ in the full momentum range, whereas that at OBELIX is 3\%-5\% of 50 to $400 \mathrm{MeV} / c$. In particular, antineutrons with momentum higher than $500 \mathrm{MeV} / c$ (about $80 \%$ of the tagged antineutrons) are unique in the world. These events can be used to study many open problems involving the physics of antineutrons, as discussed in the review [2].

Ground-state $S U(3)$ octet hyperons $\left(\Lambda, \Sigma^{+,-}, \Xi^{0,-}\right)$ and their antiparticles can be produced copiously in $J / \psi$ decays, and they can be tagged similarly to antineutrons. Since their $c \tau$ is on the order of a few centimeters, comparable to inner radius of the beam pipe (see below), only some make it to the beam pipe and/or the inner tube of the MDC, but the surviving fraction is significant. This makes the study of hyperon and antihyperon interactions with nuclei feasible. Table I lists the main $J / \psi$ decay modes relevant for hyperon/antihyperon production, together with their $c \tau$, to be compared with the geometry of the BESIII beam pipe and the MDC inner tube. A rough estimate of the available tagged particles in the $1 \times 10^{10} \mathrm{~J} / \psi$ event sample can be made according to the tag efficiency $(\sim 20 \% \div 40 \%$, depending on multiplicity of final-state particles), the $\beta \gamma$ of the produced hyperons, and their lifetimes. The numbers of particles reaching the beam pipe (as target material at BESIII) are listed in Table I.

The $\Omega^{-}$and its antiparticle $\bar{\Omega}^{+}$cannot be produced in $J / \psi$ decays due to their high mass, but they are accessible through $\psi(2 S) \quad$ decays. As $\quad \mathcal{B}\left[\psi(2 S) \rightarrow \Omega^{-} \bar{\Omega}^{+}\right]=$ $5.2 \times 10^{-5}$, they can be studied with the BESIII $\psi(2 S)$ data sample [18]. There could also be other $\psi(2 S)$ decay modes with $\Omega^{-}$or $\bar{\Omega}^{+}$in the final state, such as $\psi(2 S) \rightarrow$ $K^{-} \Xi^{0} \bar{\Omega}^{+}+$c.c. These, too, are listed in Table I, together with the number of $\Omega$ 's reaching the beam pipe in the $3 \times 10^{9} \psi(2 S)$ event data sample [18]. Of course, the $\psi(2 S)$ data sample will be able to supply more decay modes for all these hyperons and antihyperons, including the decays from the secondary charmonium states $\left[\chi_{c J}\right.$ $(J=0,1,2), J / \psi]$ produced in $\psi(2 S)$ radiative and hadronic transitions [42]. These new decay modes will broaden the momentum range of the source particles.

The BESIII $J / \psi$ data sample has been collected already, so we do not have an opportunity to gather data with a custom-made target. However, the detector material close to the interaction point in the inner detector serves as an effective target, allowing us to carry out a substantial study with the collected antineutrons.

The BESIII [18] beam pipe is $1000 \mathrm{~mm}$ long with an inner diameter of $63 \mathrm{~mm}$ and an outer diameter of $114 \mathrm{~mm}$. The central part of the beam pipe is $296 \mathrm{~mm}$ long with two layers of beryllium. The inner wall that maintains the ultrahigh vacuum in the beam pipe is $0.8 \mathrm{~mm}$ thick, and the outer beryllium wall is $0.6 \mathrm{~mm}$ thick. A $0.8 \mathrm{~mm}$ channel between the two walls is used for circulating the cooling fluid (high-purity mineral oil). Outside of the beam pipe is the MDC. The MDC consists of an outer chamber and an inner chamber, which are joined together at the end plates, sharing a common gas volume. The inner tube of the MDC is made of $1.2 \mathrm{~mm}$ thick carbon fiber with a radius of $59.2 \mathrm{~mm}$ that also provides some mechanical strength. The inner chamber of the MDC, together with the inner tube, can be replaced in case it is damaged by radiation. This design even allows us to put some target material into the current BESIII detector if we want to do antineutron physics in the near future, as will be discussed below.

The materials in the beam pipe and the inner tube of the MDC can be treated as targets of beryllium and carbon. 
TABLE I. Hyperon and antihyperon production in the $1 \times 10^{10} \mathrm{~J} / \psi$ or $3 \times 10^{9} \psi(2 \mathrm{~S})$ event data sample at BESIII. The yield of hyperons is the same as that of antihyperons, since particles and antiparticles are produced with the same rates in $J / \psi$ [or $\psi(2 S)$ ] decays via strong or electromagnetic interactions. $p_{\max }$ is the maximum momentum of the antihyperon, and $n_{\mathrm{BP}}^{Y}$ is the number of tagged antihyperons reaching the beam pipe; "--" means not available.

\begin{tabular}{lccccc}
\hline \hline Antihyperon & $c \tau(\mathrm{cm})$ & Decay mode & Branching fraction $\left(\times 10^{-3}\right)$ & $p_{\max }(\mathrm{MeV} / c)$ & $n_{\mathrm{BP}}^{Y}\left(\times 10^{5}\right)$ \\
\hline $\bar{\Lambda}$ & 7.89 & $J / \psi \rightarrow \Lambda \bar{\Lambda}$ & 1.89 & 1074 & 26 \\
& & $J / \psi \rightarrow p K^{-} \bar{\Lambda}$ & 0.87 & 876 & 9 \\
$\bar{\Sigma}^{-}$ & 2.40 & $J / \psi \rightarrow \Sigma^{+} \bar{\Sigma}^{-}$ & 1.50 & 992 & 4 \\
& & $J / \psi \rightarrow \Lambda \pi^{+} \bar{\Sigma}^{-}$ & 0.83 & 950 & 1 \\
$\bar{\Sigma}^{+}$ & 4.43 & $J / \psi \rightarrow \Lambda \pi^{-} \bar{\Sigma}^{+}$ & $\ldots$ & 945 & $\ldots$ \\
$\bar{\Xi}^{0}$ & \multirow{2}{*}{8.71} & $J / \psi \rightarrow \Xi^{0} \bar{\Xi}^{0}$ & 1.17 & 818 & 7 \\
& & $J / \psi \rightarrow \Xi^{-} \pi^{+} \bar{\Xi}^{0}$ & $\ldots$ & 685 & $\ldots$ \\
$\bar{\Xi}^{+}$ & 4.91 & $J / \psi \rightarrow \Xi^{-} \bar{\Xi}^{+}$ & 0.97 & 807 & 3 \\
& & $J / \psi \rightarrow \Xi^{0} \pi^{-} \bar{\Xi}^{+}$ & $\ldots$ & 686 & $\ldots$ \\
$\bar{\Omega}^{+}$ & 2.46 & $\psi(2 S) \rightarrow \Omega^{-} \bar{\Omega}^{+}$ & 0.05 & 774 & 0.05 \\
& & $\psi(2 S) \rightarrow K^{-} \Xi^{0} \bar{\Omega}^{+}$ & $\cdots$ & 606 & $\cdots$ \\
\hline \hline
\end{tabular}

Since all $\bar{n}$ interactions with these materials occur in the central part of the detector, all the final-state particles can be detected by the full detector, just like those produced in the primary $J / \psi$ decays, in terms of tracking, particle identification, interaction vertex determination, photon reconstruction, and so on. A schematic diagram of $e^{+} e^{-} \rightarrow J / \psi \rightarrow p \pi^{-} \bar{n}$, followed by $\bar{n}$ interaction with a proton in the beam pipe material is shown in Fig. 2. The charged pions and the photons from neutral pion decays are shown for a possible process $\bar{n} p \rightarrow \pi^{+} \pi^{+} \pi^{-} \pi^{0}, \pi^{0} \rightarrow \gamma \gamma$.

The good momentum resolution of the BESIII detector makes it possible to pinpoint the interaction vertex with a resolution of about $1 \mathrm{~mm}$ [44], so as to determine whether an antineutron interacted with beryllium or carbon, but the resolution is not sufficient to separate interaction with the inner or outer wall of the beam pipe, nor with the mineral

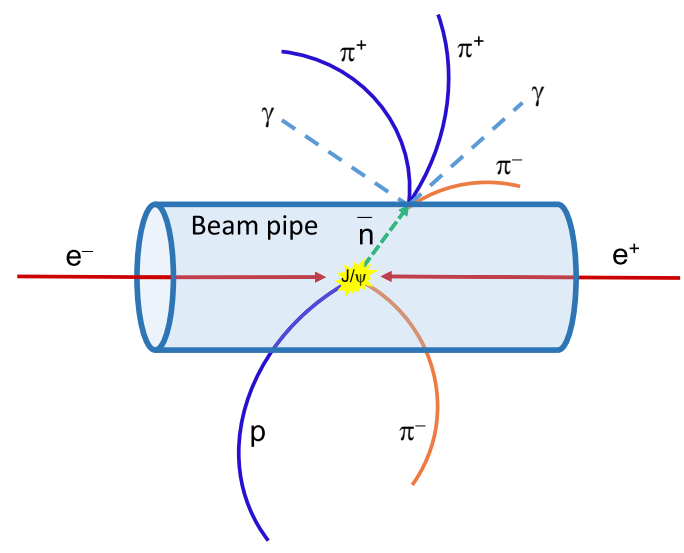

FIG. 2. Schematic diagram of $e^{+} e^{-} \rightarrow J / \psi \rightarrow p \pi^{-} \bar{n}$, followed by $\bar{n}$ interaction with a proton in the beam pipe material, $\bar{n} p \rightarrow \pi^{+} \pi^{+} \pi^{-} \pi^{0}, \pi^{0} \rightarrow \gamma \gamma$. oil between the two layers of beryllium. The background from direct $J / \psi$ decays is low, since all the final-state particles are reconstructed, and the total energy of the event is larger than the background $e^{+} e^{-} \mathrm{CM}$ energy. The background from particle interaction with target material, but with final states different from the one under study, can be suppressed thanks to the good particle identification and good momentum/energy resolution of the detector.

One can use the materials' budget to estimate the fraction of antineutrons interacting with specific components of the inner detector. Assuming an average of a $100 \mathrm{mb}$ cross section per $\bar{n} p$ or $\bar{n} n$ collision, we expect $1 \%-2 \%$ of the tagged antineutrons to interact with beryllium and another $1 \%-2 \%$ with carbon fiber targets. These are equivalent to about 100000 events each for $\bar{n}+\mathrm{Be}$ and $\bar{n}+\mathrm{C}$ interactions, respectively.

Accelerators at the tau-charm energy region with luminosity 100 times higher than current ones are being proposed, focusing on a variety of physics associated with strong and electroweak interactions. Both STCF [20] and SCTF [21] operate in a way similar to BEPCII. The detectors will be even better than BESIII in terms of tracking, particle identification, and photon detection. Experiments at these new facilities will be able to accumulate $10^{12} \mathrm{~J} / \psi$ events in one year's running time-i.e., 100 times more than the total dataset accumulated by BESIII.

If one were to simply rescale the numbers in previous sections accordingly, one would immediately see that such a machine provides a superb opportunity for physics with baryon and antibaryon sources discussed above. In fact, the case is even more striking, since several additional features in the accelerator and the detector design will result in significant improvements in the quality of both the sources and the targets. 
In the current BEPCII accelerator, the energy spread at the $J / \psi$ peak is $0.9 \mathrm{MeV}$. Since the natural width of $J / \psi$ is only about $90 \mathrm{keV}$, the peak cross section for $J / \psi$ production will be significantly increased once the beam energy spread is further reduced. It is expected that use of the monochromator scheme will significantly reduce the energy spread and increase the peak cross section, by a factor between a few times and orders of magnitude [45-47].

The range of physics topics investigated with these baryons and antibaryons can be expanded through the use of custom-made targets that can be designed and placed inside the detector, just outside of the beam pipe. The radius of the beam pipe can be made smaller than that at BEPCII, allowing the targets to be located closer to the interaction point, enlarging the acceptance of the target material and reducing the fraction of hyperon decays. In principle, one can design a barrel with several different types of materials as targets, allowing simultaneous study of different interactions. This will be extremely efficient, since all studies share the same environment and systematic effects can be investigated once for all. Of course, removable targets will be helpful for even more studies. This does not involve technical difficulties, as the current BESIII [18] design has a replaceable inner drift chamber, and the inner tube of the chamber has been used as a target in the data taking.

In order to produce baryon and antibaryon particles with higher momenta, one can use asymmetric $e^{+} e^{-}$beams which will produce $J / \psi$ in motion. Alternatively, one can utilize similar decay modes of $\psi(2 S)$, or both. One may also broaden the momentum spectra of baryons produced in $J / \psi$ decays, by utilizing $J / \psi$ boosted through a large crossing angle collision [47].

Detector design can be optimized to enhance the detection of the final states specific to the reactions discussed in the present work. The current detector design is optimized for measurements of light particles such as $e$, $\mu, \pi, K, p$, and photons. A special-purpose subdetector can be devised for the identification of particles, like deuterons, tritons, and even heavier nuclei.

In summary, we demonstrate that $J / \psi$ mesons produced in high-luminosity $e^{+} e^{-}$annihilation can provide large numbers of baryon and antibaryon particles as sources for novel nuclear and particle physics studies. These sources include all the long-lived baryons and antibaryons, especially antineutrons, hyperons $\Lambda, \Sigma^{+,-}, \Xi^{0,-}$, and their antiparticles, as well as smaller, but still large fluxes of $\Omega^{-}$ and $\bar{\Omega}^{+}$. By placing specific custom-made targets in a BESIII-like detector, one can perform a rich variety of experiments in nuclear and particle physics. Besides those discussed in the paper or listed in Table I, other decay modes with antineutrons or hyperons can also be used as sources of these particles. Although the tagging efficiency may be low in multiparticle final states, more low-momentum source particles can be produced in these modes.
With the existing $1 \times 10^{10} \mathrm{~J} / \psi$ event data sample accumulated at BESIII, many studies of the interactions of these long-lived baryons with the material in the beam pipe and the inner tube of the MDC detector can already be performed. With minor modifications of the accelerator and detector designed for STCF [20] and SCTF [21], the resulting $J / \psi$ sample can serve as a superb source of all kinds of long-lived baryons and antibaryons, opening a new era for novel highprecision nuclear and particle physics studies.

Traditional setups need to produce many different kinds of beams for different dedicated experiments and need to share accelerator time among them. This requires large resources in terms of manpower and funding, impeding such experiments. In contrast, the approach proposed here will allow experiments with different beams at the same time, requiring no additional infrastructure and minimal further investments.

This work is supported in part by the National Key Research and Development Program of China under Contract No. 2020YFA0406300; the National Natural Science Foundation of China (NSFC) under Contracts No. 11961141012, No. 11835012, and No. 11521505; and the CAS Center for Excellence in Particle Physics (CCEPP), as well as NSFC-ISF Grant No. 3423/19. C. Z. Y. thanks Sicheng Yuan for stimulating conversations about antineutron-material interactions, and M. K. thanks Avraham Gal, Simon Eydelman, and Jon Rosner for helpful discussions.

yuancz@ihep.ac.cn marek@tauex.tau.ac.il

[1] E. Rutherford, Philos. Mag. Ser. 6 21, 669 (1911).

[2] For a review, see T. Bressani and A. Filippi, Phys. Rep. 383, 213 (2003).

[3] T. Armstrong, C. Chu, J. Clement, C. Elinon, M. Furic et al. (Brookhaven-Houston-Pennsylvania State-Rice Collaboration), Phys. Rev. D 36, 659 (1987).

[4] M. Agnello et al., Nucl. Instrum. Methods Phys. Res., Sect. A 399, 11 (1997).

[5] J. Haidenbauer, S. Petschauer, N. Kaiser, U. G. Meissner, A. Nogga, and W. Weise, Nucl. Phys. A915, 24 (2013).

[6] I. Vidaña, Proc. R. Soc. A 474, 20180145 (2018).

[7] L. Tolos and L. Fabbietti, Prog. Part. Nucl. Phys. 112, 103770 (2020).

[8] R. Engelmann, H. Filthuth, V. Hepp, and E. Kluge, Phys. Lett. 21, 587 (1966).

[9] B. Sechi-Zorn, B. Kehoe, J. Twitty, and R. A. Burnstein, Phys. Rev. 175, 1735 (1968).

[10] G. Alexander, U. Karshon, A. Shapira, G. Yekutieli, R. Engelmann, H. Filthuth, and W. Lughofer, Phys. Rev. 173, 1452 (1968).

[11] F. Eisele, H. Filthuth, W. Foehlisch, V. Hepp, and G. Zech, Phys. Lett. 37B, 204 (1971).

[12] J. A. Kadyk, G. Alexander, J. H. Chan, P. Gaposchkin, and G. H. Trilling, Nucl. Phys. B27, 13 (1971). 
[13] J. M. Hauptman, J. A. Kadyk, and G. H. Trilling, Nucl. Phys. B125, 29 (1977).

[14] J. K. Ahn et al. (KEK-PS E-251 Collaboration), Nucl. Phys. A648, 263 (1999).

[15] S. Aoki, S. Asai, S. Y. Bahk, S. H. Chung, H. Funahashi, C. H. Hahn, M. Hanabata, T. Hara, K. Hoshino, M. Ieiri et al., Nucl. Phys. A644, 365 (1998).

[16] Y. Kondo et al. (KEK-PS-E289 Collaboration), Nucl. Phys. A676, 371 (2000).

[17] J. K. Ahn, S. Aoki, K. S. Chung, M. S. Chung, H. En'yo, T. Fukuda, H. Funahashi, Y. Goto, A. Higashi, M. Ieiri et al., Phys. Lett. B 633, 214 (2006).

[18] M. Ablikim et al. (BESIII Collaboration), Nucl. Instrum. Methods Phys. Res., Sect. A 614, 345 (2010); papers in D. M. Asner et al., Int. J. Mod. Phys. A 24, Suppl. 1 (2009), https://www.worldscientific.com/toc/ijmpa/24/supp01; M. Ablikim et al. (BESIII Collaboration), Chin. Phys. C 44, 040001 (2020).

[19] Q. Qin, L. Ma, J. Wang, and C. Zhang, Conf. Proc. C 100523, 2359 (2010), IPAC-2010-WEXMH01, http://accelconf.web .cern.ch/AccelConf/IPAC10/papers/wexmh01.pdf.

[20] Z. G. Zhao, Proceedings of the "International Workshop on Physics at Future High Intensity Collider 2-7 GeV in China," University of Chinese Academy of Sciences (UCAS), Hefei, China, 2015.

[21] E. Levichev, Proceedings of the "The 9th International Workshop on Charm Physics," Novosibirsk, Russia, 2018, https://indico.inp.nsk.su/event/10/session/1/contribution/ 65/material/slides/0.pdf.

[22] E. Klempt, C. Batty, and J. M. Richard, Phys. Rep. 413, 197 (2005).

[23] J. R. Ellis, E. Gabathuler, and M. Karliner, Phys. Lett. B 217, 173 (1989).

[24] J. R. Ellis, M. Karliner, D. E. Kharzeev, and M. G. Sapozhnikov, Phys. Lett. B 353, 319 (1995).

[25] J. R. Ellis, M. Karliner, D. E. Kharzeev, and M. G. Sapozhnikov, Nucl. Phys. A673, 256 (2000).

[26] A. Gal, E. V. Hungerford, and D. J. Millener, Rev. Mod. Phys. 88, 035004 (2016).

[27] E. Botta, T. Bressani, and G. Garbarino, Eur. Phys. J. A 48, 41 (2012).

[28] J. Pochodzalla et al. (P̄ANDA Collaboration), J. Phys. Soc. Jpn. Conf. Proc. 17, 091002 (2017).

[29] J. L. Rosner, J. Phys. G 34, S127 (2007).

[30] E. Klempt and A. Zaitsev, Phys. Rep. 454, 1 (2007).

[31] G. Battistoni et al., Ann. Nucl. Energy 82, 10 (2015); T. T. Böhlen, F. Cerutti, M. P. W. Chin, A. Fassò, A. Ferrari, P. G. Ortega, A. Mairani, P. R. Sala, G. Smirnov, and V. Vlachoudis, Nucl. Data Sheets 120, 211 (2014).
[32] S. Agostinelli et al. (GEANT4 Collaboration), Nucl. Instrum. Methods Phys. Res., Sect. A 506, 250 (2003); J. Allison et al., Nucl. Instrum. Methods Phys. Res., Sect. A 835, 186 (2016).

[33] J. J. Aubert et al. (E598 Collaboration), Phys. Rev. Lett. 33, 1404 (1974).

[34] J. E. Augustin et al. (SLAC-SP-017 Collaboration), Phys. Rev. Lett. 33, 1406 (1974).

[35] P. Wang, C. Z. Yuan, X. H. Mo, and D. H. Zhang, Phys. Lett. B 593, 89 (2004).

[36] M. Ablikim et al. (BESIII Collaboration), Phys. Lett. B 791, 375 (2019).

[37] P. A. Zyla et al. (Particle Data Group), Prog. Theor. Exp. Phys. 2020, 083C01 (2020).

[38] X. Li et al., Radiat. Detect. Technol. Methods 1, 13 (2017); Y. X. Guo et al., Radiat. Detect. Technol. Methods 1, 15 (2017); P. Cao et al., Nucl. Instrum. Methods Phys. Res., Sect. A 953, 163053 (2020).

[39] M. Ablikim et al. (BES Collaboration), Phys. Rev. Lett. 97, 062001 (2006).

[40] M. Ablikim et al. (BESIII Collaboration), Phys. Rev. D 86, 052011 (2012).

[41] M. Astrua, E. Botta, T. Bressani, D. Calvo, C. Casalegno, A. Feliciello, A. Filippi, S. Marcello, M. Agnello, and F. Iazzi, Nucl. Phys. A697, 209 (2002).

[42] It should be noted that particles other than those which are the focus of this paper are also produced copiously in $J / \psi$ and $\psi(2 S)$ decays. With similar methods, $\pi^{ \pm}, K^{ \pm}, K^{0} / \bar{K}^{0}$, $p / \bar{p}$, neutron, photon, and other particles can be also be tagged (for $K_{L}$, cf. Ref. [43]). Although the corresponding samples may not be large compared with those from dedicated experiments, they are produced for free, their momentum is known with high precision, and they can be used for physics studies with the same targets and detector. In a super $J / \psi$ factory, the corresponding yields will be higher by 2 orders of magnitude or more, so in certain cases the samples may become competitive with existing data from dedicated experiments.

[43] M. Amaryan et al. (KLF Collaboration), arXiv:2008.08215.

[44] M. Xu, K. L. He, Z. P. Zhang, Y. F. Wang, J. M. Bian, G. F. Cao, X. X. Cao, S. J. Chen, Z. Y. Deng, C. D. Fu et al., Chin. Phys. C 33, 428 (2009).

[45] A. A. Avdienko, G. A. Kornyukhin, I. Y. Protopopov, A. N. Skrinsky, A. B. Temnykh, G. M. Tumaikin, and A. A. Zholents, Conf. Proc. C 830811, 186 (1983), https:// inspirehep.net/files/ae36197f04d4b3b51dc8a308c5e6c331.

[46] K. Wille and A. W. Chao, SLAC/AP-032, https://lib-extopc .kek.jp/preprints/PDF/1984/8412/8412105.pdf.

[47] V. I. Telnov, arXiv:2008.13668. 